\title{
CHARACTERISTIC EXPONENTS AND SOME APPLICATIONS TO DIFFERENTIAL EQUATIONS
}

\author{
F. S. DE BLASI AND M. A. BOUDOURIDES
}

\begin{abstract}
A generalization of the Lozinskir logarithmic norm is introduced and some applications to stability of differential equations are given.
\end{abstract}

1. Introduction. Let $A$ be a continuous linear function from a real Banach space $E$ into itself. Following Lozinskiur [8] (see also [4]) the logarithmic norm $\mu[A]$ of $A$ is defined by $\mu[A]=\lim _{h \rightarrow 0+}(\|I+h A\|-1) / h$, where $I$ denotes the identity and $\|I+h A\|=\sup _{|x|=1}|x+h A(x)|$. This notion has been used to bound solutions of differential equations and to obtain asymptotic stability $[1,3,9]$. A definition of logarithmic norm for functions $A$, which are continuous but not necessarily linear, is given by Martin [10], who presents also some applications to differential equations.

In this note we consider continuous, possibly nonlinear, functions $A$ from $E$ into itself such that $A(0)=0$. For such functions we define upper and lower characteristic exponents of order $\alpha \geq 1$, denoted by $\nu_{s}^{\alpha}[A]$ and $\nu_{i}^{\alpha}[A]$ respectively. When $A$ is linear and $E$ is a real Hilbert space, we have $\nu_{s}^{1}[A]=\mu[A]$ and $\nu_{i}^{1}[A]=-\mu[-A]$. The upper and lower characteristic exponents are related to stability properties of the differential equation

$$
x^{\prime}=A(x) .
$$

For example (Theorem 3.1), $\nu_{s}^{\alpha}[A]<0\left(\nu_{i}^{\alpha}[A]>0\right)$ implies asymptotic stability (instability) of the zero solution of (1.1). A characterization of these numbers in terms of some properties of solutions of (1.1) is also given (Theorem 3.2).

2. Characteristic exponents. Let $E$ be a real Banach space with norm $|\cdot|$. Set $S_{d}=\{x \in E|| x \mid<d\}, d>0$. Let $U \subset E$ be a nonempty open convex set containing the origin. Denote by $\mathcal{F}=\mathcal{F}(U)$ the set of continuous functions from $U$ into $E$ such that $A(0)=0$. For any $\alpha \geq 1, \mathcal{Q}_{\alpha}=\mathcal{Q}_{\alpha}(U)$ is the subset of $₹$ of all $A \in \mathcal{F}$ such that $|A|_{\mathcal{Q}_{\alpha}}=\lim \sup _{x \rightarrow 0}|A(x)| /|x|^{\alpha}<+\infty ; B_{\alpha}=B_{\alpha}(U)$ is the subset of $\mathcal{Q}_{\alpha}$ of all $A \in \mathcal{Q}_{\alpha}$ which satisfy $|A(x)| \leq L_{A}|x|^{\alpha}\left(L_{A} \geq 0\right)$, for each $x \in U$. Clearly $\mathcal{Q}_{\alpha}$ and $B_{\alpha}$ are linear spaces; $|\cdot|_{\mathcal{Q}_{\alpha}}$ is a seminorm on $\mathcal{Q}_{\alpha}$. Notice that for any $\alpha>1$, the set $\mathcal{Q}_{\alpha}$ is contained in $\mathcal{Q}_{1}$.

Definition 2.1. For each $A \in \mp$ and $x \in U$, set

$$
N[A, x]=\lim _{h \rightarrow 0+}(|x+h A(x)|-|x|) / h_{\imath} .
$$

Received by the editors May 22, 1981. This paper has been presented by the second author to the special session on "Qualitative theory of differential equations" at the 87th annual meeting of the A.M.S. in San Francisco (January 7-11, 1981).

1980 Mathematics Subject Classification. Primary 34D05, 34D20.

Key words and phrases. Characteristic exponents, logarithmic norm, differential equations, stability. 
Define for $\alpha \geq 1$

$$
\nu_{s}^{\alpha}[A]=\limsup _{x \rightarrow 0} \frac{N[A, x]}{|x|^{\alpha}}, \quad \nu_{i}^{\alpha}[A]=\liminf _{x \rightarrow 0} \frac{N[A, x]}{|x|^{\alpha}} .
$$

We call $\nu_{s}^{\alpha}[A]$ and $\nu_{i}^{\alpha}[A]$ the upper and lower characteristic exponents of order $\alpha$ of $A$ at the origin.

$N[A, x]$ exists and is finite $\left[5\right.$, p. 61]. If $A \in \mathcal{Q}_{\alpha}$, we have $-|A|_{\mathcal{Q}_{\alpha}} \leq \nu_{i}^{\alpha}[A] \leq$ $\nu_{s}^{\alpha}[A] \leq|A|_{\mathcal{Q}_{\alpha}}$ and so $\nu_{s}^{\alpha}[A]$ and $\nu_{i}^{\alpha}[A]$ are finite.

We say that the functions $A$ and $B \in \mathcal{Q}_{\alpha}$ are $\alpha$-tangent at the origin, if

$$
\left.\lim _{x \rightarrow 0}|A(x)-B(x) /| x\right|^{\alpha}=0 .
$$

This is an equivalence relation in $\mathcal{Q}_{\alpha}$. For any $A \in \mathcal{Q}_{\alpha}$, denote by $[A]$ the equivalence class which contains $A$, that is the set of all $B \in \mathcal{Q}_{\alpha}$, which are $\alpha$-tangent to $A$ at the origin. For any such $B$ we have $N[A, x] \leq N[B, x]+|A(x)-B(x)|, x \in U$, from which dividing by $|x|^{\alpha}>0$ and taking upper limits as $x \rightarrow 0$, it follows $\nu_{s}^{\alpha}[A] \leq$ $\nu_{s}^{\alpha}[B]$. Thus, interchanging $A$ and $B, \nu_{s}^{\alpha}[A]=\nu_{s}^{\alpha}[B]$. The same equality holds for lower characteristic exponents. This shows that for any $A \in \mathcal{Q}_{\alpha}, \nu_{s}^{\alpha}[A]$ and $\nu_{i}^{\alpha}[A]$ depend only on the equivalence class $[A]$, not on its particular representative. It is easy to prove that for any $A, B \in \mathcal{Q}_{\alpha}$ and $x \in U$ we have

$$
\left\{\begin{array}{l}
N[t A, x]=t N[A, x], \quad t \geq 0, \\
N[A+B, x] \leq N[A, x]+N[B, x], \\
N[A+t J, x]=N[A, x]+t|x|^{\alpha}, \quad t \in \mathbf{R}, J(x)=x|x|^{\alpha-1}, \alpha \geq 1 .
\end{array}\right.
$$

If the norm is Gâteaux differentiable, the first equality in (2.1) holds for any $t \in \mathbf{R}$.

Proposition 2.1. Let $A, B \in \mathcal{Q}_{\alpha}$. Then we have: (i) $\nu_{s}^{\alpha}[t A]=t \nu_{s}^{\alpha}[A](t \geq 0)$, (ii) $\nu_{s}^{\alpha}[A+B] \leq \nu_{s}^{\alpha}[A]+\nu_{s}^{\alpha}[B]$, (iii) $\nu_{s}^{\alpha}[A+t J]=\nu_{s}^{\alpha}[A]+t\left(t \in \mathbf{R}, J(x)=x|x|^{\alpha-1}\right)$ and (iv) $\left|\nu_{s}^{\alpha}[A]-\nu_{s}^{\alpha}[B]\right| \leq|A-B|_{\Theta_{\alpha}}$.

Proof. From (2.1) we obtain (i)-(iii). From $N[A, x] \leq N[B, x]+|A(x)-B(x)|$, dividing by $|x|^{\alpha}>0$ and taking upper limits as $x \rightarrow 0$, we have $\nu_{s}^{\alpha}[A] \leq \nu_{s}^{\alpha}[B]+$ $|A-B|_{\mathcal{Q}_{\alpha}}$; thus, interchanging $A$ and $B$, (iv) follows.

The relations (i), (iii) and (iv) in Proposition 2.1 are true, if we replace upper with lower characteristic exponents. If $E$ has norm which is Gâteaux differentiable, then $N[t A, x]=t N[A, x]\left(A \in \mathcal{Q}_{\alpha}, t \leq 0, x \in U\right)$ and so, $\nu_{s}^{\alpha}[t A]=t \nu_{i}^{\alpha}[A](t \leq 0)$. By this and $\nu_{s}^{\alpha}[-(A+B)] \leq \nu_{s}^{\alpha}[-A]+\nu_{s}^{\alpha}[-B]$, we obtain $\nu_{i}^{\alpha}[A+B] \geq \nu_{i}^{\alpha}[A]+\nu_{i}^{\alpha}[B]$.

Proposition 2.2. Let $E$ be a real Hilbert space with inner product $(\cdot, \cdot)$. For any $A \in \mathcal{Q}_{\alpha}$, we have

$$
\nu_{s}^{\alpha}[A]=\limsup _{x \rightarrow 0}(A(x), x) /|x|^{\alpha+1} \quad \text { and } \quad \nu_{i}^{\alpha}[A]=\liminf _{x \rightarrow 0}(A(x), x) /|x|^{\alpha+1} .
$$

PROOF. For $x \neq 0$ and $h>0$ sufficiently small, we have

$$
\begin{aligned}
\frac{|x+h A(x)|-|x|}{h} & =\frac{|x+h A(x)|^{2}-|x|^{2}}{h(|x+h A(x)|+|x|)} \\
& =\frac{2(A(x), x)}{|x+h A(x)|+|x|}+\frac{h|A(x)|^{2}}{|x+h A(x)|+|x|} .
\end{aligned}
$$


Letting $h \rightarrow 0+$, it follows $N[A, x]=(A(x), x) /|x|$. From this, dividing by $|x|^{\alpha}$ and taking upper and lower limits as $x \rightarrow 0$, we obtain $\nu_{s}^{\alpha}[A]$ and $\nu_{i}^{\alpha}[A]$ respectively.

Under the hypotheses of Proposition 2.2, if $A \in \mathcal{Q}_{1}(E)$, we have $\nu_{s}^{1}[A]=\sup n_{0}(A)$ and $\nu_{i}^{1}[A]=\inf n_{0}(A)$, where $n_{0}(A)$ is the local nonlinear numerical range introduced by Furi and Vignoli [6].

A function $A \in \mathcal{F}$ is called positively homogeneous of degree $\alpha>0$ if for each $x \in U, t \geq 0$ such that $t x \in U$, it satisfies $A(t x)=t^{\alpha} A(x)$.

Proposition 2.3. Let $E$ be a real Hilbert space and suppose that $A \in \mathcal{Q}_{\alpha}\left(S_{r}\right), r>$ 1 , is positively homogeneous of degree $\alpha \geq 1$. Then we have $\nu_{s}^{\alpha}[A]=\sup _{|x|=1}(A(x), x)$ and $\nu_{i}^{\alpha}[A]=\inf _{|x|=1}(A(x), x)$. If, in addition, $A$ is linear and Hermitian, $\nu_{s}^{1}[A]=$ $\lambda^{\prime \prime}=\mu[A]$ and $\nu_{i}^{1}[A]=\lambda^{\prime}=-\mu[-A]$, where $\lambda^{\prime \prime}$ and $\lambda^{\prime}$ are the greatest and smallest eigenvalues of $A$ and $\mu[A]$ is the logarithmic norm of $A$.

Proof. Since $A$ is positively homogeneous of degree $\alpha$, by Proposition 2.2 we obtain $\nu_{s}^{\alpha}[A]=\sup _{|x|=1}(A(x), x)$. Hence, if $\alpha=1$ and $A$ is linear and Hermitian, it follows $\nu_{s}^{1}[A]=\lambda^{\prime \prime}$. The equality $\lambda^{\prime \prime}=\mu[A]$ can be found in $[5, \mathrm{p} .62]$. The proof for the lower characteristic exponent is similar.

Martin [10] has introduced the following nonlinear generalization of the logarithmic norm: $\mu_{M}[A]=\lim _{h \rightarrow 0+}\left(N_{M}[I+h A]-1\right) / h$, where $A \in B_{1}=B_{1}\left(S_{d}\right)$ and $N_{M}[I+h A]=\sup _{0<|x|<d}|x+h A(x)| /|x|$. He shows that $\mu_{M}[A]$ exists and is finite, if $A$ is Lipschitzean.

Proposition 2.4. Let $A \in B_{1}$ be Lipschitzean. Then $\mu_{M}[A] \geq \nu_{s}^{1}[A]$ and, if the norm of $E$ is Gâteaux differentiable, $\nu_{i}^{1}[A] \geq-\mu_{M}[-A]$. Moreover, if $A \in \mathcal{Q}_{1}(E)$ is linear and $E$ is a real Hilbert space, we have $\nu_{s}^{1}[A]=\mu[A]$ and $\nu_{i}^{1}[A]=-\mu[-A]$.

Proof. Let $\epsilon>0$. Since

$$
\mu_{M}[A]=\lim _{h \rightarrow 0+} \sup _{0<|x|<d}(|x+h A(x)|-|x|) /(h|x|),
$$

there is $h_{0}>0$ such that, for each $0<h<h_{0}$ and $x \in S_{d}, x \neq 0$, we have

$$
(|x+h A(x)|-|x|) /(h|x|) \leq \sup _{0<|x|<d}(|x+h A(x)|-|x|) /(h|x|)<\mu_{M}[A]+\epsilon .
$$

Letting $h \rightarrow 0+$, we obtain $N[A, x]<\left(\mu_{M}[A]+\epsilon\right)|x|$, from which $\nu_{s}^{1}[A] \leq \mu_{M}[A]$ follows at once. If the norm is Gâteaux differentiable, we have $\mu_{M}[-A] \geq \nu_{s}^{1}[-A]=$ $-\nu_{i}^{1}[A]$ and so $\nu_{i}^{1}[A] \geq-\mu_{M}[-A]$. Now suppose that $A \in \mathcal{Q}_{1}(E)$ is linear and $E$ is a real Hilbert space. Let $0<\epsilon<1$. Since $A$ is continuous there is $h_{0}>0$ such that for each $0<h<h_{0}$ and $x \in E,|x|=1$, we have $|x+h A(x)|>1-\epsilon$. Then from (2.2) (under the above restrictions on $h$ and $x$ ) we obtain

$$
\begin{aligned}
(|x+h A(x)|-1) / h & =\left[2(A(x), x)+h|A(x)|^{2}\right] /(|x+h A(x)|+1) \\
& \leq\left[2(A(x), x)+h\|A\|^{2}\right] /(2-\epsilon) .
\end{aligned}
$$

Hence $\sup _{|x|=1}(|x+h A(x)|-1) / h \leq\left(2 \nu_{s}^{1}[A]+h\|A\|^{2}\right) /(2-\epsilon)$ and, letting $h \rightarrow 0+$, it follows immediately $\mu[A] \leq \nu_{s}^{1}[A]$. On the other hand $\mu[A]=\mu_{M}[A] \geq \nu_{s}^{1}[A]$, thus $\mu[A]=\nu_{s}^{1}[A]$. Since $\mu[-A]=\nu_{s}^{1}[-A]=-\nu_{i}^{1}[A]$, also the equality $\nu_{i}^{1}[A]=-\mu[-A]$ is true. This completes the proof.

We observe that in Proposition 2.4 strict inequalities can hold. For example, taking $A(x)=3 x+x^{2},-1<x<1$, one has $\mu_{M}[A]=4$ and $\nu_{s}^{1}[A]=3$. 
3. Some applications to differential equations. The characteristic exponents are related to stability properties of the zero solution of (1.1). The next more general definition allows to cover also the case of stability of a set, which is invariant for (1.1). Notice that all differential equations which we consider here are supposed to satisfy hypotheses which guarantee (local) existence and uniqueness of solutions.

Let $\Omega$ be a nonempty closed and proper subset of $E$. Set

$$
d(x, \Omega)=\inf \{|x-u| \mid u \in \Omega\}, \quad x \in E,
$$

and

$$
\Omega_{r}=\{x \in E \mid d(x, \Omega)<r\}, \quad r>0 .
$$

Let $A$ be the set of continuous functions $A: \Omega_{r} \rightarrow E$ which are bounded, that is $\sup \left\{|A(x)| \mid x \in \Omega_{r}\right\}<+\infty$, and such that $\Omega$ is positively invariant for (1.1).

DEFinition 3.1. For $A \in A$ and $x \in \Omega_{r}$, set

$$
N_{\Omega}[A, x]=\limsup _{h \rightarrow 0+}[d(x+h A(x), \Omega)-d(x, \Omega)] / h .
$$

Define for $\alpha \geq 1$

$$
\begin{aligned}
& \nu_{\Omega, s}^{\alpha}[A]=\lim _{\delta \rightarrow 0+} \sup _{0<d(x, \Omega)<\delta} \frac{N_{\Omega}[A, x]}{d(x, \Omega)^{\alpha}}, \\
& \nu_{\Omega, i}^{\alpha}[A]=\lim _{\delta \rightarrow 0+} \inf _{0<d(x, \Omega)<\delta} \frac{N_{\Omega}[A, x]}{d(x, \Omega)^{\alpha}} .
\end{aligned}
$$

If $\Omega=\{0\}$ and $A \in \mathcal{F}\left(S_{r}\right)$, we obtain $\nu_{s}^{\alpha}[A]$ and $\nu_{i}^{\alpha}[A]$. We recall that $\Omega$ is stable for (1.1), if for every $\eta>0$ there is $\delta>0$ such that each solution $x(\cdot)$ of $(1.1)$ with $d(x(0), \Omega)<\delta$ satisfies $d(x(t), \Omega)<\eta, t \geq 0$. $\Omega$ is asymptotically stable for (1.1), if it is stable and there is $\sigma>0$ such that $d(x(0), \Omega)<\sigma$ implies $\lim _{t \rightarrow+\infty} d(x(t), \Omega)=0$.

THEOREM 3.1. Let $A \in A$ be such that $-\infty<\nu_{\Omega, s}^{\alpha}[A]<0\left(\right.$ resp. $0<\nu_{\Omega, i}^{\alpha}[A]<$ $+\infty)$ for some $\alpha \geq 1$. Then $\Omega$ is asymptotically stable (resp. not stable) for (1.1).

PROOF. We prove only the statement concerning $\nu_{\Omega, s}^{1}[A]$ (the same argument can be used in the other cases). Let $\epsilon>0$ satisfy $c=\nu_{\Omega, s}^{1}[A]+\epsilon<0$ and let $0<$ $\sigma<r$ be such that $0<d(x, \Omega)<\sigma$ implies $N_{\Omega}[A, x]<c d(x, \Omega)$. We claim that any solution $x(\cdot)$ of $(1.1)$ with $d(x(0), \Omega)<\sigma$ is such that $\phi(t)=d(x(t), \Omega)<\sigma$, for each $t$ in the right maximal interval of existence of $x(\cdot)$, say $[0, \omega), \omega>0$. Suppose $\phi(t)>0$ for each $t \in[0, \omega)$. If the claim is not true, there is $t_{1}>0$ such that $0<\phi(t)<\sigma$, $t \in\left[0, t_{1}\right)$, and $\phi\left(t_{1}\right)=\sigma$. Then, denoting by $D^{+}$the right upper Dini derivative, we have for $t \in\left[0, t_{1}\right)$

$$
\begin{aligned}
& D^{+} \phi(t)= \limsup _{h \rightarrow 0+} \frac{1}{h}\left[d\left(x(t)+\int_{t}^{t+h} A(x(u)) d u, \Omega\right)-d(x(t), \Omega)\right] \\
& \leq \limsup _{h \rightarrow 0+}\left(\frac{1}{h}[d(x(t)+h A(x(t)), \Omega)-d(x(t), \Omega)]\right. \\
&\left.+\left|\frac{1}{h} \int_{t}^{t+h} A(x(u)) d u-A(x(t))\right|\right) \\
& \leq N_{\Omega}[A, x(t)] .
\end{aligned}
$$

Since $\phi(t)<\sigma, t \in\left[0, t_{1}\right)$, we have $D^{+} \phi(t)<c \phi(t)$, which implies (see [7, p. 15]) $\sigma=$ $\phi\left(t_{1}\right) \leq \phi(0) \exp \left(c t_{1}\right)<\sigma$, a contradiction. Since $A$ is bounded, a standard argument 
shows that $\omega=+\infty$. Therefore $D^{+} \phi(t)<c \phi(t), t \geq 0$, and so $\phi(t) \leq \phi(0) \exp (c t)$, $t \geq 0$. Since $\Omega$ is positively invariant for (1.1), it is easy to verify that the latter inequality is still satisfied, if $\phi(t)$ vanishes for some $t \geq 0$. Thus $\Omega$ is asymptotically stable for (1.1) and the proof is complete.

EXAMPLE 3.1. Let $A(z)=\left(-y+\lambda x\left(1-|z|^{2}\right)^{k}, x+\lambda y\left(1-|z|^{2}\right)^{k}\right)$, where $z=$ $(x, y),|z|=\left(x^{2}+y^{2}\right)^{1 / 2}, \lambda \geq 0$ and $k \geq 1$ is odd. Let $\Omega=\left\{z \in \mathbf{R}^{2}|| z \mid=1\right\}$. Suppose $|z|<1$. Then $d(z, \Omega)=1-|z|$ and, for $h>0$ small enough, $d(z+h A(z), \Omega)=1-$ $|z|\left(1+p h+q h^{2}\right)^{1 / 2}$, where $p=2 \lambda\left(1-|z|^{2}\right)^{k}$ and $q=1+\lambda^{2}\left(1-|z|^{2}\right)^{2 k}$. Thus $N_{\Omega}[A, z]=-\lambda|z|\left(1-|z|^{2}\right)^{k}$. If $|z|>1$, a similar computation furnishes $N_{\Omega}[A, z]=$ $\lambda|z|\left(1-|z|^{2}\right)^{k}$. Hence $N_{\Omega}[A, z] / d(z, \Omega)^{k}=-\lambda|z|(1+|z|)^{k},|z| \neq 1$. Therefore $\nu_{\Omega, s}^{k}[A]=\nu_{\Omega, i}^{k}[A]=-\lambda 2^{k}$.

Notice that Theorem 3.1 is valid under more general assumptions. For instance, one can prove that, if $\nu_{\Omega, s}^{1}[A]=-\infty$ then the set $\Omega$ is asymptotically stable for (1.1). A trivial example in which this happens is given by $A(x)=-x, x \in \mathbf{R}$ and $\Omega=[a, b], a<0, b>0$.

The characteristic exponents and, in particular, the logarithmic norm can be interpreted in terms of Liapunov functions. This is evident, if in Definition 2.1 we consider $|\cdot|$ as the Liapunov function $V(x) \equiv|x|$. This motivates the following generalization. Let $\mathcal{V}$ be the set of functions $V: S_{r} \rightarrow \mathbf{R}, r>0$, which are Lipschitzean and satisfy $|x| \leq V(x) \leq b|x|(b \geq 1), x \in S_{r}$. Let $A \in \mathcal{F}\left(S_{r}\right)$ and let $V \in \mathcal{V}$. For each $x \in S_{r}$, set $N[A, V, x]=\limsup _{h \rightarrow 0+}[V(x+h A(x))-V(x)] / h$. $N[A, V, x]$ is finite, since $V$ is Lipschitzean. Then, for any $\alpha \geq 1$, define $\nu_{s}^{\alpha}[A, V]=$ $\limsup _{x \rightarrow 0} N[A, V, x] / V(x)^{\alpha}$ and $\nu_{i}^{\alpha}[A, V]=\liminf _{x \rightarrow 0} N[A, V, x] / V(x)^{\alpha}$.

Let $-\infty<\nu_{s}^{\alpha}[A, V]<0$. Denote by $\Gamma_{\alpha}$ the set of numbers $c<0$ for which there is $\delta=\delta(c)>0$ such that

$$
\begin{aligned}
& \alpha=1,\left|x_{0}\right|<\delta \text { implies } V(x(t)) \leq V\left(x_{0}\right) \exp (c t), \quad t \geq 0, \\
& \alpha>1,\left|x_{0}\right|<\delta \text { implies } V(x(t))^{\alpha-1} \leq \frac{V\left(x_{0}\right)^{\alpha-1}}{1-c(\alpha-1) V\left(x_{0}\right)^{\alpha-1} t}, \quad t \geq 0 .
\end{aligned}
$$

Here $x(\cdot)$ denotes the solution of $(1.1)$ with $x(0)=x_{0}$.

REMARK 3.1. If $A \in \mathcal{Q}_{\alpha}=\mathcal{Q}_{\alpha}\left(S_{r}\right)$ and $\nu_{s}^{\alpha}[A, V]<0$, then the set $\Gamma_{\alpha}$ is nonempty and bounded from below. Consider $\alpha=1$ (the same argument can be used when $\alpha>1)$ and fix $\epsilon>0$ such that $\hat{c}=\nu_{s}^{1}[A, V]+\epsilon<0$. Since $A \in \mathcal{Q}_{1}$, there is $0<\sigma<r$ such that $|A(x)| \leq L|x|, x \in S_{\sigma}$, where $L=|A|_{\mathcal{Q}_{1}}+1$. As in the proof of Theorem 3.1 (with $\Omega=\{0\}$ ) one finds $0<\delta<\sigma$ such that any solution $x(\cdot)$ of (1.1) with $x(0) \in S_{\delta}$ remains in $S_{\sigma}$ and satisfies $V(x(t)) \leq V(x(0)) \exp (\hat{c} t), t \geq 0$. Thus $\Gamma_{1}$ is nonempty. Moreover, denoting by $K$ the Lipschitz constant of $V$, we have $\left|D^{+} V(x(t))\right| \leq K\left|x^{\prime}(t)\right| \leq K L V(x(t)), t \geq 0$, and so, $D^{+} V(x(t)) \geq-K L V(x(t))$. Therefore, for each $x(0) \in S_{\delta}$ and $t \geq 0, V(x(t)) \geq V(x(0)) \exp (-K L t)$. This implies that $\Gamma_{1}$ is bounded from below.

DEFINITION 3.2. Define $\beta_{s}^{\alpha}[A, V]$ to be the greatest lower bound of $\Gamma_{\alpha}$.

THEOREM 3.2. Let $A \in \mathcal{Q}_{\alpha}\left(S_{r}\right)$. Suppose that $V \in \mathcal{V}$ has continuous derivative in $S_{r} \backslash\{0\}$, and $-\infty<\nu_{s}^{\alpha}[A, V]<0$. Then $\nu_{s}^{\alpha}[A, V]=\beta_{s}^{\alpha}[A, V]$.

PROOF. We prove only the statement concerning $\nu_{s}^{1}[A, V]$ (when $\alpha>1$ the proof is similar). Let $\epsilon>0$ be such that $\hat{c}=\nu_{s}^{1}[A, V]+\epsilon<0$ and $0<\delta<\sigma$ correspond as in Remark 3.1. It follows that $\hat{c} \in \Gamma_{1}$ and so $\beta_{s}^{1}[A, V] \leq \nu_{s}^{1}[A, V]$. Suppose that this inequality is strict and let $c \in \Gamma_{1}$ satisfy $\beta_{s}^{1}[A, V]<c<\nu_{s}^{1}[A, V]$. There is then 
$0<\hat{\delta}<\delta$ such that any solution $x(\cdot)$ of (1.1). with $x(0) \in S_{\hat{\delta}}$ satisfies (3.1). On the other hand, from the definition of $\nu_{s}^{1}[A, V]$, it follows that if we fix $\theta>0$ such that $c<\nu_{s}^{1}[A, V]-\theta=\gamma$, there is $\hat{x} \in S_{\hat{\delta}}(\hat{x} \neq 0)$ for which $N[A, V, \hat{x}]>\gamma V(\hat{x})$. Let $x(\cdot)$ be the solution of (1.1) with $x(0)=\hat{x}$. From the hypotheses, $t \rightarrow d(V(x(t))) / d t$ exists and is continuous for $t \in[0, \hat{t}]$, for some $\hat{t}>0$. Clearly, $N[A, V, x(t)]=D^{+} V(x(t))=$ $d(V(x(t))) / d t$ and so, $N[A, V, x(t)]$ is a continuous function of $t \in[0, \hat{t}]$. Taking $\hat{t}$ smaller, if necessary, we have $N[A, V, x(t)] \geq \gamma V(x(t)), t \in[0, \hat{t}]$. Thus, $D^{+} V(x(t)) \geq$ $\gamma V(x(t))$, which implies $V(x(t)) \geq V(\hat{x}) \exp (\gamma t), t \in[0, \hat{t}]$. The latter inequality and (3.1), with $x_{0}=\hat{x}$ and $t \in[0, \hat{t}]$, imply $\gamma \leq c$, a contradiction. This completes the proof.

A similar characterization can be given for $\nu_{i}^{\alpha}[A, V]$.

For any $\alpha \geq 1$, let $\mathcal{N}_{\alpha}$ be the set of continuous functions $B: I \times S_{r} \rightarrow E, r>1$, $I=[a,+\infty), B(t, 0) \equiv 0$, such that $\limsup _{x \rightarrow 0}|B(t, x)| /|x|^{\alpha}<+\infty$ exists uniformly with respect to $t \in I$. For any $B \in \mathcal{N}_{\alpha}$, we define $N[B, x, t], \nu_{s}^{\alpha}[B, t]$ and $\nu_{i}^{\alpha}[B, t]$ as in Definition 2.1, replacing $A$ with $B$. Here we require that the upper limit $\nu_{s}^{\alpha}[B, t]$ and the lower limit $\nu_{i}^{\alpha}[B, t]$ exist uniformly with respect to $t \in I$. Notice that, for any $B \in \mathcal{N}_{\alpha}, \nu_{s}^{\alpha}[B, t]$ and $\nu_{i}^{\alpha}[B, t]$ are bounded functions of $t \in I$.

For any $B \in \mathcal{N}_{\alpha}$, consider the differential equation

$$
x^{\prime}=B(t, x) \text {. }
$$

The following proposition extends a result of Wazewski [11] (see also [12]).

PROPOSITION 3.1. Let $E$ be a separable real Hilbert space. Let $B \in \mathcal{N}_{\alpha}$ be such that, for any $t \in I, B(t, \cdot)$ is positively homogeneous of degree $\alpha \geq 1$. Then, for each $\left(t_{0}, x_{0}\right) \in I \times S_{r}$ there exists $t_{1}>t_{0}$ such that, for any $t \in\left[t_{0}, t_{1}\right]$, the solution $x(\cdot)$ of (3.2), with $x\left(t_{0}\right)=x_{0}$, satisfies

$$
\begin{gathered}
\left|x_{0}\right| \exp \left(\int_{t_{0}}^{t} \nu_{i}^{1}[B, u] d u\right) \leq|x(t)| \leq\left|x_{0}\right| \exp \left(\int_{t_{0}}^{t} \nu_{s}^{1}[B, u] d u\right), \quad \alpha=1 \\
\frac{\left|x_{0}\right|}{\left(1+(1-\alpha)\left|x_{0}\right|^{\alpha-1} \int_{t_{0}}^{t} \nu_{i}^{\alpha}[B, u] d u\right)^{1 /(\alpha-1)}} \leq|x(t)| \\
\leq \frac{\left|x_{0}\right|}{\left(1+(1-\alpha)\left|x_{0}\right|^{\alpha-1} \int_{t_{0}}^{t} \nu_{s}^{\alpha}[B, u] d u\right)^{1 /(\alpha-1)}}, \quad \alpha>1 .
\end{gathered}
$$

PROOF. As in the Proposition 2.3 one obtains $\nu_{s}^{\alpha}[B, t]=\sup _{|x|=1}(B(t, x), x)$ and $\nu_{i}^{\alpha}[B, t]=\inf _{|x|=1}(B(t, x), x), t \in I$. From this, since the unit sphere of $E$ is separable, it follows that $\nu_{s}^{\alpha}[B, t]$ and $\nu_{i}^{\alpha}[B, t]$ are (bounded) measurable functions of $t \in I$. Thus the integrals in the statement of the proposition make sense. Let now $\alpha=1$. Let $t_{1}>t_{0}$ such that $|x(t)|<r, t \in\left[t_{0}, t_{1}\right]$. Suppose $x(t)>0, t \in\left[t_{0}, t_{1}\right]$. Since $D^{+}|x(t)|=(B(t, x(t)), x(t)) /|x(t)|$ and $B(t, \cdot)$ is positively homogeneous, we have $\nu_{i}^{1}[B, t]|x(t)| \leq D^{+}|x(t)| \leq \nu_{s}^{1}[B, t]|x(t)|$, which implies (3.3). When $x(t)=0$ for some $t \in\left[t_{0}, t_{1}\right]$, then by uniqueness of solutions $x(t) \equiv 0$ and (3.3) is trivially satisfied. When $\alpha>1$, the proof is similar.

For further properties concerning differential equations with homogeneous righthand side, see Busenberg and Jaderberg [2]. The next proposition generalizes a result of Brauer [1]. 
PROPOSITION 3.2. Let $E$ be a separable real Hilbert space. If, for some $\alpha \geq 1$, $B \in \mathcal{N}_{\alpha}$ and $\lim \sup _{t \rightarrow+\infty}\left(\int_{a}^{t} \nu_{s}^{\alpha}[B, u] d u\right) /(t-a)<0$, then the origin is asymptotically stable for (3.2).

The proof is omitted since it is similar to that of Brauer. We notice only that, as in the proof of Proposition 3.1, $\nu_{s}^{\alpha}[B, t]$ turns out to be a bounded measurable function of $t \in I$ and so the integral in the statement makes sense.

\section{REFERENCES}

1. F. Brauer, Perturbations of nonlinear systems of nonlinear equations, J. Math. Anal. Appl. 14 (1966), 198-206.

2. S. N. Busenberg and L. K. Jaderberg, Decay characteristics for differential equations without linear terms, J. Differential Equations 18 (1975), 87-102.

3. W. A. Coppel, Stability and asymptotic behavior of differential equations, Heath, Boston, Mass., 1965.

4. G. Dahlquist, Stability and error bounds in the numerical integration of ordinary differential equations, Kungl. Tekn. Högsk. Handl. Stockholm, No. 130 (1959).

5. Ju. L. DaleckiY and M. G. KreYn, Stability of solutions of differential equations in Banach spaces, Math. Mono., No. 43, Amer. Math. Soc., Providence, R.I., 1974.

6. M. Furi and A. Vignoli, Spectrum for nonlinear maps and bifurcation in the non-differentiable case, Ann. Mat. Pura Appl. (4) 113 (1977), 265-285.

7. V. Lakshmikantham and S. Leela, Differential and integral inequalities, theory and applications, Vol. I, Academic Press, New York, 1969.

8. S. M. Lozinski1, Error estimates for numerical integration of ordinary differential equations. I, Izv. Vyšs. Ǔebn. Zaved. Matem., No. 5, (6) (1958), 52-90. (Russian)

9. R. H. Martin, Jr., A bound for solutions of Volterra-Stieltjes integral equations, Proc. Amer. Math. Soc. 23 (1969), 506-512.

10. $\ldots$, Bounds for solutions of a class of nonlinear differential equations, J. Differential Equations 8 (1970), 416-430.

11. T. Wazewski, Sur la limitation des intégrales des systèmes d'équations differentielles linéaires ordinaires, Studia Math. 10 (1948), 185-213.

12. A. Wintner, Asymptotic integration constants, Amer. J. Math. 68 (1946), 553-559.

Istituto Matematico “U. Dini”, Viale Morgagni 67/A, 50134 Firenze, Italy

Department of Mathematics, Democritus University of Thrace, Xanthi, GREECE 